\title{
Systems biology of diuretic resistance
}

\author{
Mark A. Knepper
}

Epithelial Systems Biology Laboratory, National Heart, Lung, and Blood Institute, NIH, Bethesda, Maryland, USA.

\begin{abstract}
Diuretics are commonly used to treat hypertension and extracellular fluid volume expansion. However, the development of compensatory responses in the kidney limits the benefit of this class of drugs. In this issue of the $J C l$, Grimm and colleagues use a systems biology approach in mice lacking the kinase SPAK and unravel a complex mechanism that explains thiazide diuretic resistance. The overall process involves interactions among six different cell types in the kidney.
\end{abstract}

\section{Systems biology-based approaches for complex phenotypes}

The "art" in any scientific study is in asking the right questions. Although the experimental process and interpretation of experimental data are highly systematized, the process of hypothesis generation often does not follow a standard course. The problem of hypothesis generation is particularly critical when studying animal models of disease. While it is easy enough to delete a particular gene in mice that is believed to be associated with a human disease, specific gene knockouts routinely result in phenotypic features in the mouse that could not have been predicted because of the high levels of complexity that result from the interaction of processes in multiple organs, tissues, and cell types. Once an animal model is generated and functional phenotypes are recognized, how can the investigator formulate a set of questions that, if answered, are likely to lead to an understanding of the phenotype and the corresponding human disease processes? This is where systems biology-based approaches can be highly effective. In this issue, Grimm and colleagues (1) provide an excellent example of the application of systems biology tools to better understand the phenotype observed in an animal model of disease. These studies by Grimm et al. reveal a complex mechanism that involves interactions among at least six different renal cell types that can explain acquired resistance to thiazide diuretics.

\section{Compensatory mechanisms in} the kidney: a systems approach The study by Grimm and colleagues investigates key elements of renal function critical to extracellular fluid volume homeostasis and blood pressure control. The kidney regulates these variables through the control of $\mathrm{Na}$ and $\mathrm{Cl}(\mathrm{Na}-\mathrm{Cl})$ excretion $(2,3)$ by regulating the transport of these ions in the renal tubule. Several renal tubule segments contribute to $\mathrm{Na}-\mathrm{Cl}$ reabsorption, each using different transporter proteins (Table 1). The resulting redundancy of transport regulation provides robust control of extracellular fluid volume and blood pressure.

Three of the apical sodium transporters highlighted in Table 1 are the molecular targets for the major classes of diuretics: loop diuretics, which act in the thick ascending limb of Henle's loop; thiazide diuretics, which act in the distal convoluted tubule (DCT); and amiloride-like compounds, which act on the principal cells (PCs) of the collecting duct. These agents are frequently used in the treatment of extracellular fluid volume excess or hypertension. However, their efficacy tends to be compromised over time by the development of diuretic resistance. It

Related Article: p. 2136

Conflict of interest: The author has declared that no conflict of interest exists.

Reference information: J Clin Invest. 2015;125(5):1793-1795. doi:10.1172/JCI81505.

is well established that diuretic resistance is a result of compensatory increases in sodium transport in segments of the renal tubule other than that targeted by a particular diuretic compound $(4,5)$, although it is not clear how these adaptations are regulated. Grimm and colleagues (1) investigated potential mechanisms involved in this compensatory response using a mouse model in which the gene encoding the protein kinase SPAK (Stk39) has been deleted (herein referred to as SPAK KO mice). SPAK is necessary for activation of the thiazide-sensitive $\mathrm{Na}-\mathrm{Cl}$ cotransporter (NCC). The absence of SPAK can be viewed as analogous to the state that results from chronic thiazide treatment. Despite markedly diminished $\mathrm{Na}-\mathrm{Cl}$ transport in the DCT, SPAK KO mice only manifest a very mild salt-losing phenotype, presumably as a result of compensatory $\mathrm{Na}-\mathrm{Cl}$ transport elsewhere in the kidney. Grimm and colleagues invoked a discovery approach that started with expression microarray studies to find out how a compensatory response develops in SPAK KO mice. These experiments allowed determination of which transcripts are increased or decreased in the renal cortices of SPAK KO mice relative to WT controls. Instead of focusing on individual genes, Grimm and colleagues concentrated on groups of related genes that appeared to be coregulated in SPAK KO mice. It should be noted that because of multiple hypothesis testing, false-positive responses for individual genes are likely; however, simultaneous responses of multiple related genes are unlikely to be an artifact, owing to the multiplicative nature of probabilities. The most useful gene groupings were those that were generated by mapping the responding genes to the cells in which they were expressed, using data from single-nephron transcriptomics (6). This mapping allowed Grimm et al. to recognize that multiple renal epithelial cell types are involved in the compensation for loss of NCC function and allowed them to formulate a set of mechanistic hypotheses that could be addressed with focused experimentation. The outcome of 
Table 1. Tubule segments that reabsorb $\mathrm{Na}$ and $\mathrm{Cl}$ in the renal cortex

\begin{tabular}{|c|c|c|c|}
\hline Segment & Cell type & Apical salt transporter (gene symbol) & Diuretics-targeting segment \\
\hline PT & PT cell & $\begin{array}{l}\mathrm{Na}-\mathrm{H} \text { exchanger } 3(\mathrm{~S} / \mathrm{c} 9 \mathrm{a} 3) \text {, Na-phosphate } \\
\text { cotransporter } 2 \text { (S/c34a1) }\end{array}$ & None \\
\hline CTAL & CTAL cell & NKCC2 (SIc12a1) & Loop diuretics, e.g., bumetanide \\
\hline DCT & DCT cell & NCC (SIc12a3) & Thiazides \\
\hline CNT & CNT cell & ENaC (Scnn1a, Scnn1b, Scnn1g) & Amiloride and congeners \\
\hline CNT & $\alpha-I C$ & None & None \\
\hline CNT & $\beta-I C$ & $\begin{array}{c}\text { Pendrin (Slc26a4), Na-dependent Cl-bicarbonate } \\
\text { exchanger (SIc4a8) }\end{array}$ & None \\
\hline CCD & CD PC & $\mathrm{ENaC}($ Scnn1a, Scnn1b, Scnn1g) & Amiloride and congeners \\
\hline CCD & $\alpha-I C$ & None & None \\
\hline CCD & $\beta-I C$ & $\begin{array}{c}\text { Pendrin (S/c26a4), Na-dependent Cl-bicarbonate } \\
\text { exchanger (SIc4a8) }\end{array}$ & None \\
\hline
\end{tabular}

CTAL, cortical thick ascending limb; NKCC2 , Na-K-Cl cotransporter.

their systems biology-based approach is an integrative model that is summarized in Figure 1.

\section{Systems biology: new technology and old logic}

What is "systems biology"? Although multiple definitions have been offered, a reasonable one for the present purposes is "The study of a biological process in which all of the relevant components are studied together in parallel to discover integrative mechanisms." Grimm and colleagues used oligonucleotide microarrays in their study, although there is a plethora of additional methodologies that allow multiple parallel analyses. For example, the performance characteristics of modern mass spectrometers allow investigators to unveil all or most of the proteome of a given cell type or tissue (7) and to carry out global assessment of the properties of proteins, such as global phospho-proteomics (8) and protein half-life profiling across the proteome (9). Likewise, recently developed instrumentation for deep DNA sequencing (10) allows investigators to pursue

Figure 1. Compensation for $\mathrm{Na}-\mathrm{Cl}$ transport loss in the DCT involves changes in multiple renal cell types. Using a systems biology approach, Grimm and colleagues (1) concluded that multiple renal cell types are involved in the compensatory response to thiazides. The identified cell types include DCT cells (green), $\alpha$-ICs (gray), CNT cells, CCD PCs (CNT cells and CCD PCs, coral), PT cells (yellow), and $\beta-$ ICs (blue). Together, adaptive responses in these cells coordinate an integrated compensatory network in the kidney. See ref. 1 for details. ECF, extracellular fluid. an astonishing number of questions at a genome-wide level. This includes the application of RNA-seq for comprehensive transcriptomics and ChIP-seq to carry out

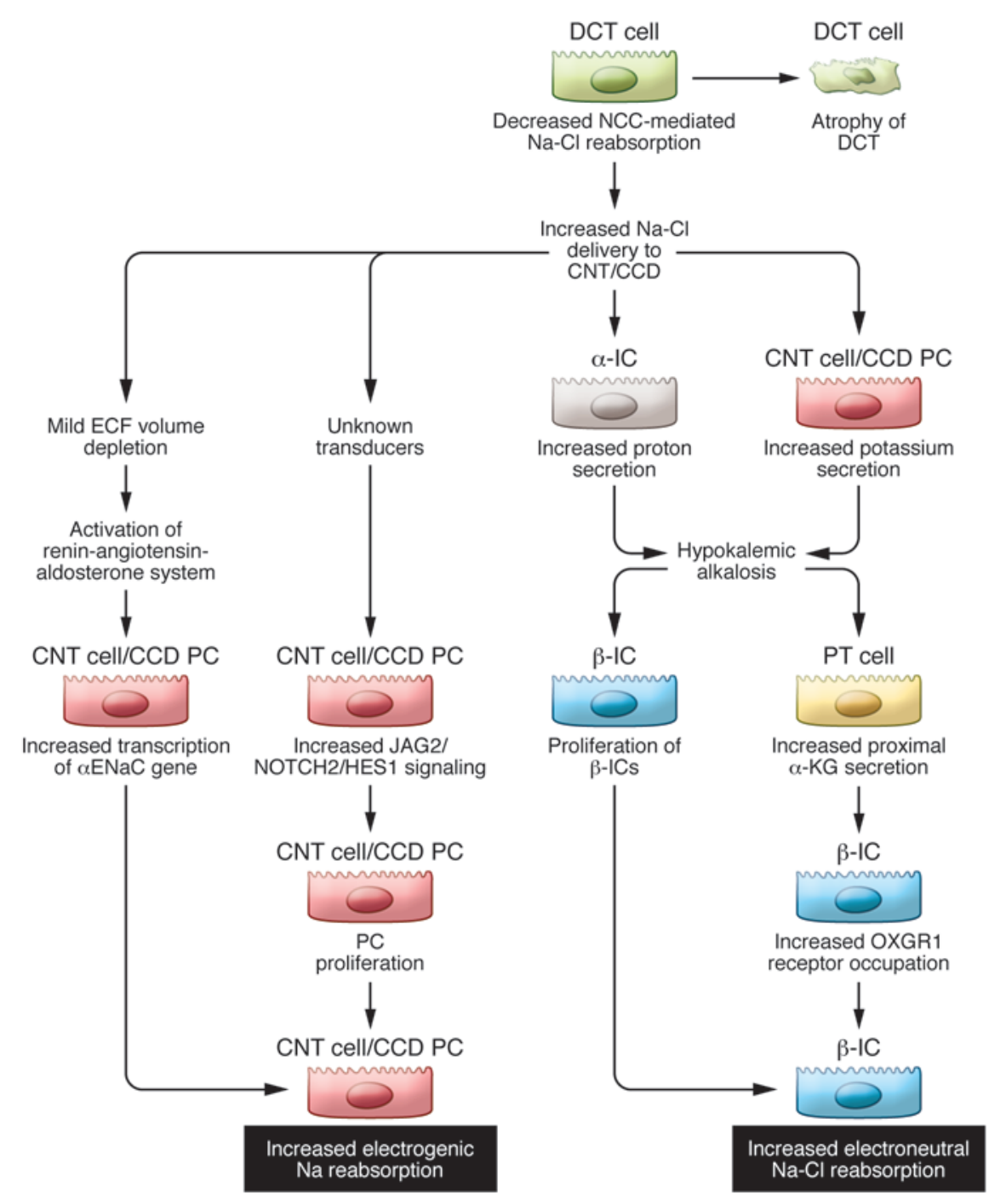

genome-wide mapping of various epigenetic modifications and the binding of specific proteins such as transcription factors and RNA polymerase II. The expanded use of these systems-level approaches over the past ten years has been abetted by the development of better computational tools, including facile software tools like those exploited by Grimm and colleagues.

Investigators must negotiate an important conceptual barrier in order to take advantage of the new systems biology tools. As illustrated by Grimm and colleagues, systems biology does not start with a specific hypothesis. Rather, there is a general question that is addressed in an openended fashion. This thinking process is not unlike the standard "differential diagnosis" approach that has been used in medicine for many decades. Here, the starting point is the patient with a chief complaint, a set of symptoms, some physical findings, and 
some standard laboratory results. These data are all collected without hypothesis and can be thought of as analogous to the results of a transcriptomic profiling study like the one reported by Grimm et al. The standard approach in medical diagnosis is to organize the data in a logical manner and make a list of possible diagnoses that are consistent with the patient's chief complaint, leading to the generation of the differential diagnosis list. Matching the patterns in the data to expectations, based on the physician's knowledge of disease processes, allows prioritization of all the possible diagnoses. The analogous step in the systems approach of Grimm and colleagues was their classification of affected genes into logical groups that point to specific mechanistic hypotheses. After going through their list, physicians can then order additional studies that would best differentiate among possible diagnoses, a process akin to hypothesis-driven experimentation in the laboratory. Thus, although the ability to gather and manage large data sets is new, the epistemological rationale for systems biology is not. In fact, the logical approach inherent to both systems biology and the differential diagnosis method in medicine was described in the nineteenth century by John Stuart Mill (11). Mill called this approach the "Method of Agreement," which he contrasted with the "Method of Difference," which ultimately came to be known as the hypothesis-driven approach.

\section{New pathophysiologic insights and future directions}

As summarized in Figure 1, changes in six different renal cell types account for the compensatory response to loss of NCC function in SPAK KO mice. Grimm and colleagues determined that two $\mathrm{Na}-\mathrm{Cl}$ transport processes are upregulated in these animals: epithelial sodium channeldependent (ENaC-dependent) electrogenic transport in the PCs of the connecting tubule and cortical collecting ducts (CNT/CCD) (12) and the pendrin-dependent electroneutral $\mathrm{Na}-\mathrm{Cl}$ reabsorption process in the $\beta$-intercalated cells ( $\beta$-ICs) of the same segments (13). Moreover, Grimm et al. demonstrated that upregulation of both processes is largely the result of remodeling of the CNT/CCD to produce an increase in the numbers of PCs and $\beta$-ICs, at the expense of $\alpha$-intercalated cells $(\alpha-\mathrm{ICs})$. Moreover, Grimm and colleagues provided data that are compatible with a role for NOTCH signaling in the observed PC hyperplasia and also demonstrated the involvement of the $G$ proteincoupled receptor OXGR1 in $\beta$-ICs, which is known from previous studies to bind $\alpha$-ketoglutarate $(\alpha-K G)$ and stimulate electroneutral $\mathrm{Na}-\mathrm{Cl}$ transport (14). Finally, the authors demonstrated that a coordinated set of gene expression changes in the proximal tubule (PT) appears to account for an increase in the urinary abundance of $\alpha$-KG. These findings, guided by the microarray results, were all solidified through focused experiments. For example, direct ion transport measurements in isolated perfused CCDs revealed a six-fold increase in $\mathrm{Cl}$ absorption in tubules microdissected from SPAK KO mice relative to that observed in WT controls.

The study by Grimm et al. provides an impressive amount of new information that is relevant to the treatment of hypertension and extracellular fluid volume expansion syndromes. Although there are some conceptual holes in the model, such as how JAG2/NOTCH/HES1 signaling is induced, it provides a beginning point for the development of new therapeutic agents that can enhance the efficacy of diuretics by damping the compensatory response. Several elements of the demonstrated network could become drug targets, although the obvious ones would be the transporter molecules involved in electroneutral $\mathrm{Na}-\mathrm{Cl}$ absorption across $\beta$-ICs (Table 1 ).

\section{Acknowledgments}

M.A. Knepper is supported by the National Heart Lung and Blood Institute's Intramural Research Program (HL006129, HL006128, and HL001285).
Address correspondence to: Mark A. Knepper, National Institutes of Health, 10 Center Dr., Bldg 10, Room 6N260, Bethesda, Maryland 20892-1603, USA. Phone: 301. 496.3064; E-mail: knep@helix.nih.gov.

1. Grimm PR, et al. Integrated compensatory network is activated in the absence of NCC phosphorylation. J Clin Invest. 2015;125(5):2136-2150.

2. Guyton AC. Blood pressure control--special role of the kidneys and body fluids. Science. 1991;252(5014):1813-1816.

3. Crowley SD, et al. Angiotensin II causes hypertension and cardiac hypertrophy through its receptors in the kidney. Proc Natl Acad Sci US A. 2006;103(47):17985-17990.

4. Kaissling B, Bachmann S, Kriz W. Structural adaptation of the distal convoluted tubule to prolonged furosemide treatment. Am J Physiol. 1985;248(3 pt 2):F374-F381.

5. $\mathrm{Na} \mathrm{KY,} \mathrm{et} \mathrm{al.} \mathrm{Upregulation} \mathrm{of} \mathrm{Na}^{+}$transporter abundances in response to chronic thiazide or loop diuretic treatment in rats. Am J Physiol Renal Physiol. 2003;284(1):F133-F143.

6. Cheval L, et al. Atlas of gene expression in the mouse kidney: new features of glomerular parietal cells. Physiol Genomics. 2011;43(3):161-173.

7. Beck M, Claassen M, Aebersold R. Comprehensive proteomics. Curr Opin Biotechnol. 2011;22(1):3-8.

8. Rinschen MM, et al. Quantitative phosphoproteomic analysis reveals vasopressin V2receptor-dependent signaling pathways in renal collecting duct cells. Proc Natl Acad Sci U S A. 2010;107(8):3882-3887.

9. Sandoval PC, Slentz DH, Pisitkun T, Saeed F, Hoffert JD, Knepper MA. Proteome-wide measurement of protein half-lives and translation rates in vasopressin-sensitive collecting duct cells. J Am Soc Nephrol. 2013;24(11):1793-1805.

10. Metzker ML. Sequencing technologies - the next generation. Nat Rev Genet. 2010;11(1):31-46.

11. Mill JS. System of Logic Ratiocinative and Inductive. Honolulu, Hawaii, USA: University Press of the Pacific; 1891.

12. Rossier BC, Baker ME, Studer RA. Epithelial sodium transport and its control by aldosterone: the story of our internal environment revisited. Physiol Rev. 2015;95(1):297-340.

13. Leviel F, et al. The $\mathrm{Na}^{+}$-dependent chloridebicarbonate exchanger SLC4A8 mediates an electroneutral $\mathrm{Na}^{+}$reabsorption process in the renal cortical collecting ducts of mice. JClin Invest. 2010;120(5):1627-1635.

14. Tokonami N, et al. $\alpha$-Ketoglutarate regulates acid-base balance through an intrarenal paracrine mechanism. JClin Invest. 2013;123(7):3166-3171. 